\title{
A NECESSARY AND SUFFICIENT CONDITION FOR ORDERS IN DIRECT SUMS OF COMPLETE SKEWFIELDS TO HAVE ONLY FINITELY MANY NONISOMORPHIC IN- DECOMPOSABLE INTEGRAL REPRESENTATIONS
}

BY K. W. ROGGENKAMP

Communicated by Irving Reiner, May 15, 1969

Let $K$ be an algebraic number field with ring of integers $R$. For an $R$-order $\Lambda$ in the semisimple $K$-algebra $A$ it seems to be one of the most important problems-from the viewpoint of integral representations - to characterize those orders $\Lambda$, for which the number $n(\Lambda)$ of nonisomorphic indecomposable $\Lambda$-lattices is finite. This problem is far from having a satisfying solution. However, a breakthrough came at the end of 1967, when Drozd-Roiter [3] and Jakobinski [5] gave, independently of each other, a necessary and sufficient condition for the finiteness of $n(\Lambda)$, in case $\Lambda$ is commutative. Whereas Jakobinski's methods seem to be restricted to the commutative case, the methods of Drozd-Roiter bear the possibilities of a generalization to the noncommutative case. This note shall be a small contribution in that direction: We shall give here a necessary and sufficient condition for the finiteness of $n(\Lambda)$ in case $\Lambda$ is an order in a direct sum of skewfields over a $\mathcal{P}$-adic number field. We shall first fix the notation and then sketch the proof of our theorem; a more explicit version is going to be published later (cf. [6], [7]).

$R$ : a complete discrete rank one valuation ring with finite residue class field,

$K$ : the quotient field of $R$,

$D_{i}: 1 \leqq i \leqq n$ : finite dimensional separable skewfields over $K$, $A=\sum_{i}^{n} \oplus D_{i}$,

$\Gamma$ : the unique maximal $R$-order in $A$,

$\Lambda:$ an $R$-order in $A$,

$N=\operatorname{rad}(\Lambda)$ : the Jacobson radical of $\Lambda$,

${ }_{\Lambda} \Re^{f}$ : the category of finitely generated unitary left $\Lambda$-modules, ${ }_{\Lambda} \mathcal{P}^{f}:$ the category of the projective modules in ${ }_{\Lambda} \mathbb{T}^{f}$,

${ }_{\Delta} \mathfrak{T T}^{0}$ : the category of $\Lambda$-lattices; i.e., $M \in_{\Lambda} \mathfrak{T}^{f}$ with $M \in \in_{R} \mathcal{Q}^{f}$, $n(\Lambda)$ : the number of nonisomorphic indecomposable $\Lambda$-lattices, $\mu_{\Lambda}(X)$ : the minimal number of generators of $X \in{ }_{\Lambda} M^{f}$, $\operatorname{rad}_{\Lambda}(X)$ : the intersection of the maximal left $\Lambda$-submodules of $X \in{ }_{\Lambda} \mathbb{T}^{f}$. 
Homomorphisms are written opposite to the scalars.

(1) THEOREM. $n(\Lambda)$ is finite if and only if

(i) $\mu_{\Lambda}(\Gamma / \Lambda) \leqq 2$ and

(ii) $\mu_{\Lambda}\left(\operatorname{rad} \Lambda_{\Lambda}(\Gamma / \Lambda)\right) \leqq 1$.

SkETCH OF THE PROOF. The theorem is easily reduced to the case where $\Lambda$ is indecomposable as left $\Lambda$-module. For the proof of the necessity, a technique of Dade [1] is used, which allows us to construct an infinite number of nonisomorphic indecomposable $\Lambda$-lattices in case one of the conditions in (1) is violated:

(2) Lemma. Let $\Lambda_{1}$ be an $R$-order in $A$ containing $\Lambda$ such that $N \Lambda_{1}$ is a two-sided $\Lambda_{1}$-ideal, and put $a=\Lambda_{1} / N \Lambda_{1}, \Re=\Lambda / N$. If for each $n \in N$, there exists a left $\mathcal{K}$-submodule $v_{n}$ of $Q^{(n)}$, the direct sum of $n$ copies of $a$, such that

(i) $a v_{n}=Q^{(n)}$,

(ii) for an idempotent $\pi \in \operatorname{End}_{Q}\left(Q^{(n)}\right)$ with $\left.\pi\right|_{v_{n}}: v_{n} \rightarrow v_{n}$, we have $\pi=0$ or $\pi=1$, then $n(\Lambda)=\infty$.

For the necessity of the proof of (1) we set up $v_{n}$ in the following form:

$$
\begin{aligned}
v_{n}=\left\{x_{1}+y_{1} \alpha, x_{2}+y_{2} \alpha+y_{1} \beta, x_{3}+y_{3} \alpha+y_{2} \beta,\right. & \cdots, x_{n} \\
& \left.+y_{n} \alpha+y_{n-1} \beta\right\}
\end{aligned}
$$

where $\alpha$ and $\beta$ are fixed elements in $\propto$ and $x_{i}, y_{i}, 1 \leqq i \leqq n$, are arbitrary elements in $\mathcal{K}$. Then $v_{n}$ is a left $\mathscr{K}$-module (we remark, that the elements in $\mathscr{K}$ do not necessarily commute with $\alpha$ or $\beta$ ). If $1, \alpha, \beta$ are linearly independent (from the left) over $\mathcal{K}$, then a $\mathcal{K}$-basis of $v_{n}$ is given by $\left\{e_{i}\right\}_{1 \leqq i \geqq n}, e_{i}=\left(0, \cdots, 0,1_{i}, 0, \cdots, 0\right)$, and $\left\{f_{i}\right\}_{1 \leqq i \leqq n}$, $f_{i}=\alpha e_{i}+\beta e_{i+1}, e_{n+1}=0$. Then $v_{n}$ satisfies (i) of (2).

$\phi \in \operatorname{End}_{\alpha}\left(Q^{(n)}\right)$ can be represented by an $(n \times n)$-matrix $\left(\phi_{i j}\right)$ with entries in $Q$. The condition $\left.\phi\right|_{v_{n}}: v_{n} \rightarrow v_{n}$ implies

$\phi_{i j}\left(e_{i}\right)=x_{i j}+y_{i j} \alpha+y_{i, j-1} \beta ; \quad i, j=1, \cdots, n, y_{i 0}=0, x_{i j}, y_{i j} \in \Re$, and from the requirement $\phi\left(f_{i}\right) \in v_{n}$ we obtain the following system of linear equations:

$$
\begin{gathered}
\alpha x_{i j}+\alpha y_{i j} \alpha+\alpha y_{i, j-1} \beta+\beta x_{i+1, j}+\beta y_{i+1, j} \alpha+\beta y_{i+1, j-1} \beta=a_{j}^{i}+b_{j}^{i} \alpha+b_{j-1}^{i} \beta, \\
a_{j}^{i}, b_{j}^{i} \in K ; i, j=1, \cdots, n, y_{n+1, j}=0, x_{n+1, j}=0 .
\end{gathered}
$$

Now it is clear, how we proceed in the proof of the necessity of (1). One shows first, that only the two cases 
(i) $\mu_{\Lambda}(\Gamma / \Lambda) \geqq 3$ and

(ii) $\mu_{\Lambda}(\Gamma / \Lambda)=2, \mu_{\Lambda}\left(\operatorname{rad}_{\Lambda}(\Gamma / \Lambda)\right)=2$ can occur.

For (i) we choose, for the application of (2), $\Lambda_{1}=\Gamma$; i.e., $Q=\Gamma / N \Gamma$, and in (ii) we take $\Lambda_{1}=\Lambda_{0}=\Lambda+N \Gamma$; i.e., $Q=\Lambda_{0} / N \Lambda_{0}$. The goal is to show that in $Q$ there exist $\alpha$ and $\beta$ such that the solutions of (4) imply $\phi=1$ or $\phi=0$, if $\phi \in \operatorname{End}_{a}\left(Q^{n}\right)$ is idempotent. To do so, we have to know the products $\bar{\alpha} x, \bar{\alpha} x \bar{\alpha}, \bar{\alpha} x \beta, \beta x \bar{\alpha}, \beta x \beta$, for $x \in \mathcal{K}$, in terms of the left $\mathscr{K}$-basis $\left\{e_{i}\right\}_{1 \leq i \leqq n},\left\{f_{i}\right\}_{1 \leq i \leq n}$ of $v_{n}$. The computational treatment of the 16 different cases shows that (2) can be applied.

The proof of the sufficiency of (1) is even more computational than the previous part. The central role in this part of the proof is played by so-called "Bass-orders"; an $R$-order $\Lambda^{\prime}$ in $A$ is called a Bass-order if every $R$-order $\tilde{\Lambda}$ in $A$, containing $\Lambda^{\prime}$ is a Gorenstein-order; i.e., $\operatorname{Hom}_{R}\left(\tilde{\Lambda}_{\tilde{\Lambda}}, R\right)$ is a generator in $\tilde{\Lambda}_{\boldsymbol{\Lambda}} \mathfrak{f}^{f}$. The importance of Bass-orders $\Lambda^{\prime}$ for the proof of (1) originates from the fact, that for every $M \in_{\Lambda^{\prime}}, T^{0}$ we have $M \in_{\tilde{\Lambda}} \rho^{f}$ for an $R$-order $\tilde{\Lambda} \supset \Lambda^{\prime}$; in particular, $n\left(\Lambda^{\prime}\right)<\infty$.

The conditions (i) and (ii) of (1) imply immediately, that $A$ is the direct sum of at most three skewfields (always under the condition, that $\Lambda$ is indecomposable as left $\Lambda$-module). Thus we have to distinguish the following three cases:

(5) (i) $A$ is a skewfield,

(ii) $A$ is the direct sum of two skewfields,

(iii) $A$ is the direct sum of three skewfields.

The crucial point in the proof of the sufficiency of (1) is, that we may assume:

$$
N^{-1}=\{a \in A \mid N a \subset \Lambda\}, \quad N=\operatorname{rad}(\Lambda),
$$

is a Bass-order and $\mu_{\Lambda}(\Gamma / \Lambda)=2$.

The cases in (5) have to be treated separately:

In $(5, \mathrm{i})$ we associate with $M \in{ }_{\Lambda} \mathscr{T}^{0}$ the exact sequence

$$
0 \rightarrow N M \rightarrow M \rightarrow M / N M \rightarrow 0 ;
$$

here $N M \in_{N^{-1}} \mathfrak{I T}^{0}$ is a lattice over the Bass-order $N^{-1}$.

Since $\Lambda / N=K_{K}$ is a field, we have $M / N M \cong \mathscr{K}^{(m)}$ for some $m \in N$.

In $(5, \mathrm{ii})$ we associate with $M \in{ }_{\Lambda} \mathfrak{T}^{0}$ the exact sequence

$$
0 \rightarrow M \cap A e_{1} M \rightarrow M \rightarrow M /\left(M \cap A e_{1} M\right) \rightarrow 0,
$$

where $e_{1}$ is a primitive idempotent in $A$, such that $\Lambda\left(1-e_{1}\right)$ is a maximal $R$-order and $\Lambda e_{1}$ is a Bass-order. Thus $M \cap A e_{1} M \in_{\Lambda e_{1}} \mathfrak{T l}^{0}$ and $M /\left(M \cap A e_{1} M\right) \cong \Lambda\left(1-e_{1}\right)^{m}$ for some $m \in N$.

In $(5, \mathrm{iii})$ we associate with $M \in{ }_{\Lambda} \mathfrak{T}^{0}$ the exact sequence 


$$
0 \rightarrow M \cap A\left(1-e_{3}\right) M \rightarrow M \rightarrow M /\left(M \cap A\left(1-e_{3}\right) M\right) \rightarrow 0,
$$

where $e_{3}$ is a primitive idempotent in $A$, such that $\Lambda e_{3}$ is a maximal order and $\Lambda\left(1-e_{3}\right)$ is a Bass-order. Thus $\left.M \cap A\left(1-e_{3}\right) M \in_{\Lambda\left(1-e_{3}\right)}\right)^{0}$ and $M /\left(M \cap A\left(1-e_{3}\right) M\right) \cong \Lambda e_{3}^{(m)}$ for some $m \in N$.

This indicates the direction for a possible proof of the sufficiency of (1): In each of the above cases, we can associate with $M \in_{\Lambda} \mathscr{T K}^{0}$ an exact sequence

$$
E: 0 \rightarrow M^{\prime} \rightarrow M \rightarrow M^{\prime \prime} \rightarrow 0,
$$

where $M^{\prime}$ is a lattice over some Bass-order $\Lambda_{1}$. Thus

$$
M^{\prime} \cong \sum_{i=1}^{s} \oplus M_{i}^{\prime}{ }^{\left(s_{i}\right)}
$$

where $M_{i}^{\prime} \neq M_{j}^{\prime}$ for $i \neq j$, and $M_{i}^{\prime} \in \in_{\Lambda_{\rho}} \rho^{f}$ for an $R$-order $\Lambda_{\rho} \supset \Lambda_{1}$. In addition, $M^{\prime}$ is a characteristic submodule of $M$ and $\operatorname{Hom}_{\Lambda}\left(M^{\prime \prime}, M^{\prime}\right)$ $=0$. Moreover, $M^{\prime \prime} \cong S^{(m)}$, where $S \in{ }_{\Lambda} \mathscr{T}^{f}$ is a ring such that the Krull-Schmidt theorem is valid for $\Lambda$-direct summands of $M^{\prime \prime}$.

Thus, the equivalence class $[E]$ of the exact sequence $E$ lies in

$$
\begin{aligned}
\operatorname{Ext}_{\Lambda}^{1}\left(S^{(m)}, \sum_{i=1}^{n} \oplus M_{i}^{\prime\left(s_{i}\right)}\right) \stackrel{\text { nat. }}{\cong} \sum_{i=1}^{s} \oplus \operatorname{Ext}_{\Lambda}^{1}\left(S^{(m)}, M_{i}^{\prime\left(s_{i}\right)}\right) \stackrel{\text { nat. }}{\cong} \\
\sum_{i=1}^{s} \oplus\left(\operatorname{Ext}_{\Lambda}^{1}\left(S, M_{i}^{\prime}\right)\right)_{m \times s_{i}},
\end{aligned}
$$

and we may associate, in a unique fashion, with the isomorphism class of $M$ an $\left(m \times \sum_{i=1}^{s} s_{i}\right)$-matrix

$$
X_{M}=\left(X_{1}, \cdots, X_{s}\right), \quad X_{i} \in\left(\operatorname{Ext}_{\Lambda}^{1}\left(S, M_{i}^{\prime}\right)\right)_{m X_{i} \cdot} .
$$

But $\operatorname{Ext}_{\Lambda}^{1}\left(M^{\prime \prime}, M^{\prime}\right)$ is an $\left[\operatorname{End}_{\Lambda}\left(M^{\prime \prime}\right)\right.$, $\left.\operatorname{End}_{\Lambda}\left(M^{\prime}\right)\right]$-bimodule; observe, that homomorphisms are written opposite to the scalars, and the elements in $\operatorname{End}_{\Lambda}\left(M^{\prime \prime}\right)$ may be represented as $(m \times m)$-matrices $Z$, and the elements in $\operatorname{End}_{\Lambda}\left(M^{\prime}\right)$ may be represented as $\left(\sum_{i=1}^{s} s_{i}\right.$, $\left.\sum_{i=1}^{s} s_{i}\right)$-matrices $\boldsymbol{Y}$. Hence we can form the extensions $\boldsymbol{Z} \boldsymbol{X}_{M} \boldsymbol{Y}$. Under the above hypotheses, we may apply a lemma of HellerReiner [4], which reduces the decomposition of $M$ to the decomposition of matrices:

(6) $M$ is decomposable if and only if $Z X_{M} Y$ decomposes as matrix, where $Z \in \operatorname{End}_{\Lambda}\left(M^{\prime \prime}\right)$ and $Y \in \operatorname{End}_{\Lambda}\left(M^{\prime}\right)$ are invertible matrices.

These ideas lead the way for the computational part of the proof of (1); the following steps have to be taken:

1. Find all $R$-orders $\Lambda_{\rho}$, that contain the Bass-order $\Lambda_{1}$ (there are only finitely many). 
2. For every $\rho$ find all nonisomorphic indecomposable projective $\Lambda_{\rho}$-lattices $M_{i_{\rho}}$ (there are only finitely many).

3. Compute $\operatorname{Ext}_{\Lambda}^{1}\left(S, M_{i_{p}}\right)$ explicitly.

4. Compute $\operatorname{Hom}_{\Lambda}\left(M_{i_{\rho}}, M_{j_{\rho}}\right)$ explicitly.

5. Compute how $\operatorname{Hom}_{\Lambda}\left(M_{i_{\rho}}, M_{j_{\rho}}\right)$ acts on

$$
\operatorname{Ext}_{\Lambda}^{1}\left(S, M_{i_{\rho}}\right) \rightarrow \operatorname{Ext}_{\Lambda}^{1}\left(S, M_{j_{\rho}}\right),
$$

and how $\operatorname{End}_{\Lambda}(S)$ acts on $\operatorname{Ext}_{\Lambda}^{1}\left(S, M_{i_{p}}\right)$.

6. Characterize the matrices $X \in \sum_{i=1}^{s} \oplus\left(\operatorname{Ext}_{\Lambda}^{1}\left(S, M_{i}^{\prime}\right)\right)_{m \times s_{i}}$, which actually do correspond to exact sequences of the type $E$.

7. Decompose these matrices under $Z X Y$, where $Z$ and $Y$ are invertible.

8. Show, that the number of nonequivalent indecomposable ones among these matrices is finite.

\section{REFERENCES}

1. E. C. Dade, Some indecomposable group representations, Ann. of Math. (2) 77 (1963), 406-412.

2. Ju. A. Drozd and A. V. Roiter, Commutative rings with a finite number of integral indecomposable representations, Izv. Akad. Nauk SSSR 31 (1967), 783-798. (Russian)

3. Ju. A. Drozd, V. V. Kirichenko and A. V. Roiter, On hereditary orders and Bass orders, Izv. Akad. Nauk SSSR 31 (1967), 1415-1436. (Russian)

4. A. Heller and I. Reiner, Representations of cyclic groups in rings of integers. I, II, Ann of Math. (2) 76 (1962), 73-92; (2) 77 (1963), 318-328.

5. H. Jakobinski, Sur les ordres commutatifs avec un nombre fini de réseaux indécomposables, Acta Math. 118 (1967), 1-31.

6. K. W. Roggenkamp, Orders in sums of $P_{\text {-adic skewfields, with an infinite number }}$ of integral representations, MS 1968.

7. - Charakterisierung von Ordnungen in einer direkten Summe kompletter Schiefkörper, die nur endlich viele nicht isomorphe unzerfällbare Darstellungen haben, Habilitationsarbeit, Gieben, 1969.

University of Montreal, Montreal, Quebec, Canada 